This is a postprint version of the following published document:

Artero-Guerrero, J. A.; Pernas-Sánchez, J.; López-Puente, J.; Varas, D. (2014). "On the influence of filling level in CFRP aircraft fuel tank subjected to high velocity impacts, Composite Structures". Composite Structures, v. 107, January, pp. 570-577. DOI: 10.1016/j.compstruct.2013.08.036

Proyectos:

DPI2010-15123

CCG10-UC3M/DPI-4694.

(C) Elsevier

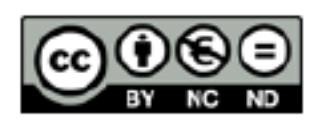

This work is licensed under a Creative Commons Attribution-NonCommercialNoDerivatives 4.0 International License. 


\title{
On the influence of filling level in CFRP aircraft fuel tank subjected to high velocity impacts
}

\author{
J.A. Artero-Guerrero, J. Pernas-Sánchez, J. López-Puente, \\ D.Varas * \\ Department of Continuum Mechanics and Structural Analysis. University Carlos \\ III of Madrid. Avda. de la Universidad, 30. 28911 Leganés, Madrid, Spain
}

Key words: Carbon fiber; Hydrodynamic RAM; fluid-structure interaction; partially fluid-filled tank; impact; numerical simulation.

\begin{abstract}
In this work, the process of impact that takes place in a partially filled tank is analyzed, performing a numerical simulation, in order to understand the response of the composite laminated structure. The commercial finite-element code LS-DYNA v.R7 has been used to simulate an Hydrodynamic RAM event created by a steel spherical projectile impacting a partially water-filled woven CFRP square tube using two different approaches (MM-ALE and SPH). The intralaminar and interlaminar damage have been taken into account implementing an user subroutine and by * Corresponding author. Fax number: 34 916248331. E-mail address:
dvaras@ing.uc3m.es
\end{abstract}


means of a cohesive interaction, respectively. Once the numerical model is validated using available experimental data, the effect of the filling level in the failure of the tank is analyzed in detail taking advantage of the information provided by the numerical model.

\section{Introduction}

The importance of laminated composites structures is increasing everyday in the aeronautical and aerospace industries. The special characteristics of this kind of materials (high strength-to-weight and stiffness-to-weight ratios as well as an anisotropic behavior) make them specially useful to optimize designs fulfilling the requirements of those mentioned industries, reducing weight and hence saving fuel consumption. The carbon fiber reinforced plastics (CFRP), commonly manufactured with an epoxy matrix, are one of the most used composite materials for structural applications, such as the fuselage and wings. The successful usage of these materials in primary structures depends on the understanding of their response to a range of potential impact loadings and resulting damage mechanisms.

Vulnerability studies of CFRP aerospace structures are becoming an issue of great importance in the design of any aircraft [1]. These structures may suffer different types of high velocity impact loads. Bird strikes [2] or hailstones [3] are specially dangerous because of their high possibility of occurrence and their disastrous consequences. The ice released from the edge of a propeller blade may impact the nacelle of the twin engine or the fuselage [4], and runway debris may impact the underside of the wing structures [5] causing hydrodynamic ram (HRAM) effects in the fuel tanks. 
The HRAM phenomenon, which is considered one of the most important factors in aircraft vulnerability, appears when a high-energy object penetrates a fluid-filled tank and transfers its kinetic energy through the fluid to the surrounding structure. This effect increases the risk of catastrophic failure and excessive structural damage. HRAM is particularly dangerous for aircrafts with lightweight designs, and hence with composite wing fuel tanks, because the structural resistance of their integral fuel tanks cannot be improved by strengthening the airframe since it would counteract the requirements of a lightweight design. Vulnerability to HRAM has been usually related to military aircraft, but commercial airplanes are not exempt of its effect. Maybe the most well-known example of the importance of the HRAM phenomenon is the Concorde accident that occurred in 2000. The final investigation report revealed that the HRAM had played a significant role in the aircraft failure.

Usually, the HRAM phenomenon is analyzed considering completely filled tanks; but in real flight situation, it is more likely that an impact may occur when the tank is partially filled. The influence of the filling percentage on the failure of the tanks has been clearly shown in experimental tests [6]. The HRAM effects in a partially filled tank can not be neglected with respect to the effects observed in a completely filled one. Indeed the failure induced in a partially filled tank can be so dangerous as in a completely filled one.

The process of impact that takes place in a partially filled tank should be carefully analyzed in order to understand the response of the composite laminated structure. To achieve this, an impact of an steel sphere into a partially water-filled CFRP woven laminate tank, reproducing an HRAM event, is simulated in this work. The intralaminar damage of the CFRP has been taken into account implementing an user subroutine. The interlaminar damage is reproduced using a cohesive interaction. Two different approaches (MM-ALE and SPH) have been used in order to demonstrate if both methods can accurately reproduce such a complex phenom- 
enon in a partially filled tank. Once the numerical model is validated by means of the available experimental data, the effect of the filling level in the failure of the tank can be analyzed in detail taking advantage of the information provided by the numerical model.

\section{Definition of the problem}

The problem configuration detailed in a previous work of D. Varas et al. [6] has been chosen in order to take advantage of the experimental results obtained and hence being able to validate the numerical model. The tests consisted on high velocity impacts of steel spheres into a CFRP woven tank filled at different water levels.

The impacted tanks consisted on square woven CFRP tubes $150 \mathrm{~mm}$ wide, $2.2 \mathrm{~mm}$ thick and $750 \mathrm{~mm}$ long. The composite woven laminated selected was the AGP193-PW manufactured by Hexcel Composite, composed by 10 plies $\left([0]_{10}\right)$. Each ply was made with a plain weave of AS4 carbon fibers and the 8552 epoxy resin. The tube was closed with two PMMA windows $30 \mathrm{~mm}$ thick, fixed with four steel bars, which allow the recording of the whole impact and penetration process by means of a Photron Ultima APX-RS digital high-speed camera. An Arrisun 12 Plus lamphead with a 1200W Hydrargyrum Medium Arc Iodide (HMI) lamp was used to assure an appropriate lighting and obtain optimal images of the penetration process. Pressure in two different points of the fluid was obtained by pressure transducers (PCB 138A06). The position of the pressure gauges, near from the impact point (PTn) and far from the impact point (PTf), can be seen in Fig. 1.

The projectile that impacts into the CFRP tubes consists in $12.5 \mathrm{~mm}$ diameter steel sphere, accelerated with a one stage light gas gun, capable of storing gas at a maximum pressure of 300 bar. Two different impact velocities were performed: 600 and $900 \mathrm{~m} / \mathrm{s}$. The CFRP woven tanks were filled with water at different volume 


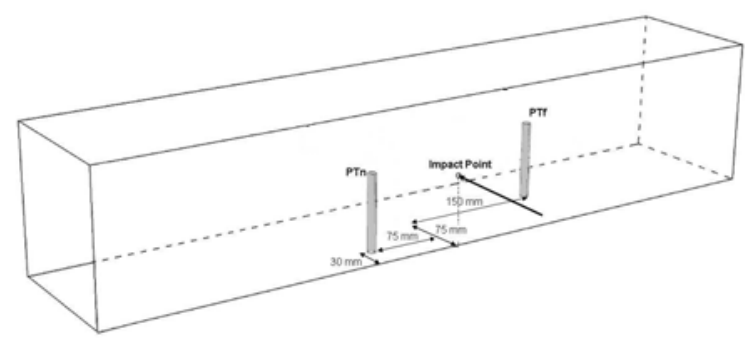

Fig. 1. Sketch of the CFRP tube instrumented.

fractions: 60,75 and $100 \%$. In this paper, only conditions corresponding to partially filled tanks (60 and $75 \%$ ) will be considered for the simulations in order to focus on the results and the analysis of such an special cases.

\section{Numerical implementation}

The nature of the HRAM phenomenon makes really complex to use a lagrangian method to reproduce the problem considered. In cases where deformation is extremely large, as it occurs in a fluid, the mesh gets distorted leading to numerical problems (drop in explicit time step, worsening in results accuracy, error termination of simulations...). In a previous work [7], the authors used a Coupled Eulerian Lagrangian method implemented in ABAQUS/Explicit to reproduce the HRAM phenomenon occurred in completely fluid filled CFRP tubes subjected to high velocity impacts. As it has been already mentioned the experimental works performed [6] show that failure in partially fluid filled CFRP cases is not negligible compared to completely filled cases. The tube wall that initially is not in contact with water suffers the impact of a layer of fluid created by the cavity, and the deformation and failures that appear in this wall depend not only in the projectile velocity, but also in the kinetic energy of that layer of fluid. Therefore, it is important to accurately model the fluid and the structure to reproduce that specific event and its effect. In this work, the commercial finite element software LS-DYNA v.R7 is used. This 
software provides several techniques to solve fluid-structure interaction; particularly two approaches have been used to model the fluid behavior: multimaterial Arbitrary Lagrangian Eulerian (MM-ALE) and the Smooth Particle Hydrodynamics (SPH). The lagrangian technique has been used to discretise the surrounding structure and the projectile.

\subsection{MM-ALE fluid model}

The MM-ALE technique allows the motion of the mesh independently on the fluid flow, avoiding mesh distortion problems. The multimaterial approach permits to define more than one material inside each element. Fig. 2 shows both the whole domain of the ALE model and the fluids considered to reproduce a partially filled case. The problem under consideration can be simplified attending its symmetry, hence only half of the problem has to be modeled obtaining a desirable reduction of the computational cost. The domain dimensions have been defined to assure that the deformed CFRP tube walls never reach the boundary of the MM-ALE domain. Therefore, the interaction between fluid and the surrounding structure is computed during the whole simulation, being able of reproducing the effect of the HRAM phenomenon. The whole MM-ALE mesh is discretised by means of 8 node solids elements, multimaterial, with reduced integration and hourglass control. In order to assure an accurate contact between fluid and the surface of the woven CFRP tube walls, both parts were discretized with the same element size. This fact avoids possible leakage problems [8]. The element size of the fluid mesh was chosen according to previous studies regarding deceleration of an sphere inside a fluid; a value of $2 \mathrm{~mm}$ has been selected. Finally, 708.806 MM-ALE elements have been used in the model. 


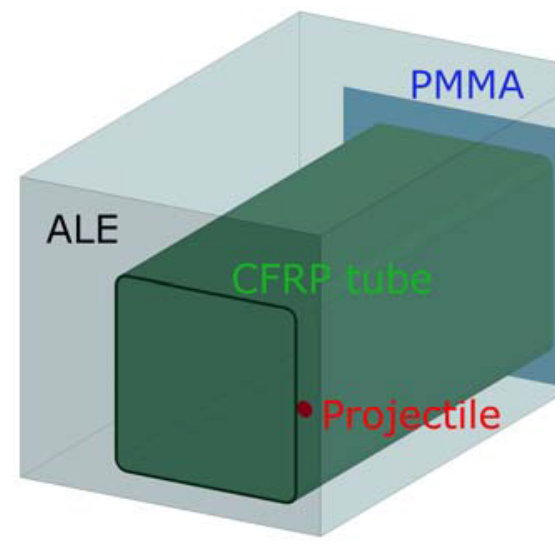

(a) MM-ALE domain

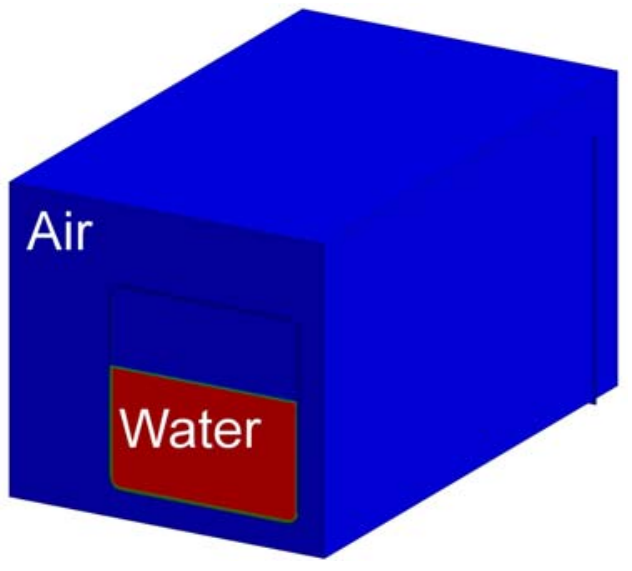

(b) MM-ALE fluids

Fig. 2. MM-ALE model done in LS-DYNA v.R7.

The fluid material behavior is defined by the following viscous constitutive equation:

$$
\sigma=2 \eta \dot{\varepsilon}^{\prime}-P I
$$

where $\eta$ is the dynamic viscosity, $\dot{\varepsilon}^{\prime}$ is the deviatoric strain rate tensor, $P$ is the pressure and I, the identity tensor. The pressure $P$ is related with density $\rho$ using a Mie-Grüneisen equation of state, where:

$$
P=\frac{\rho_{0} \mu D^{2}\left[1+\left(1-\frac{\gamma_{0}}{2}\right) \mu-\frac{a}{2} \mu^{2}\right]}{\left[1-\left(S_{1}-1\right) \mu-S_{2} \frac{\mu^{2}}{\mu+1}-S_{3} \frac{\mu^{3}}{(\mu+1)^{2}}\right]^{2}}+\left(\gamma_{0}+a \mu\right) E
$$

for compressed materials and $P=0$ for expanded materials, avoiding negative pressure in the fluid. In equation 2 , the pressure $P$ is calculated as a function of the compression $\mu=\rho / \rho_{0}-1$, where $\rho$ and $\rho_{0}$ are the current and the initial density respectively, and of the internal energy per unit volume E. $S_{1}, S_{2}$ and $S_{3}$ are coefficients of the slope of the $u_{s}-u_{p}$ curve where $u_{s}$ is the shock velocity and $u_{p}$ is the particle velocity. $D$ is the intercept of the $u_{s}-u_{p}$ curve, which corresponds to the adiabatic sound speed of water. $\gamma_{0}$ is a material constant called Grüneisen gamma and $a$ is the first volume correction to $\gamma_{0}$. Water properties (table 1) are obtained from [9].

The air was modelled using the same constitutive equation 1 , but in this case a 
Linear Polynomial Equation of State is used. Pressure now is defined as:

$$
P=C_{0}+C_{1} \mu+C_{2} \mu^{2}+C_{3} \mu^{3}+\left(C_{4}+C_{5} \mu+C_{6} \mu^{2}\right) E
$$

The air is considered as an ideal gas by setting $C_{0}=C_{1}=C_{2}=C_{3}=C_{6}=0$ and $C_{4}=C_{5}=\gamma-1$ where $\gamma=\frac{C_{p}}{C_{v}}=1.4$ is the ratio between the specific heats. The properties used for air are defined in table 1 .

\begin{tabular}{c|cc|cccccc|cc} 
& $\rho_{0}\left(\mathrm{~kg} / \mathrm{m}^{3}\right)$ & $\eta(P a \cdot s)$ & $D(\mathrm{~m} / \mathrm{s})$ & $S_{1}$ & $S_{2}$ & $S_{3}$ & $\gamma_{0}$ & $a$ & $C_{4}$ & $C_{5}$ \\
\hline \hline Water & 1000 & $0.89 \cdot 10^{-3}$ & 1448 & 1.979 & 0 & 0 & 0.11 & 3.0 & - & - \\
Air & 1.22 & $1.77 \cdot 10^{-5}$ & - & - & - & - & - & - & 0.4 & 0.4
\end{tabular}

Table 1

Water and air parameters used in the simulation for MM-ALE and SPH models.

\subsection{SPH fluid model}

$\mathrm{SPH}$ is a mesh-free method where the fluid is discretised by means of particles. Particles are not jointed by connectivity constraints, indeed each particle must search its neighbours and interacts with them through a smoothing kernel method. Since no mesh is defined, distortion problems can not occur. The SPH method requires a large number of particles uniformly distributed to provide reasonable results [10]. Several particle sizes were analyzed in order to achieve an optimal mesh density; a particle diameter of $2.98 \mathrm{~mm}$ was selected obtaining 197.125 and 248.125 particles for the $60 \%$ and $75 \%$ fluid filled volume cases respectively. In the SPH model no air has to be defined, since the particles can move freely in any direction deforming the tank walls (Fig. 3). The constitutive law and the equation of state used to model the water behaviour is the same as in the ALE method, described in the previous section. The fluid-structure interaction is achieved by means of a coupling algorithm 


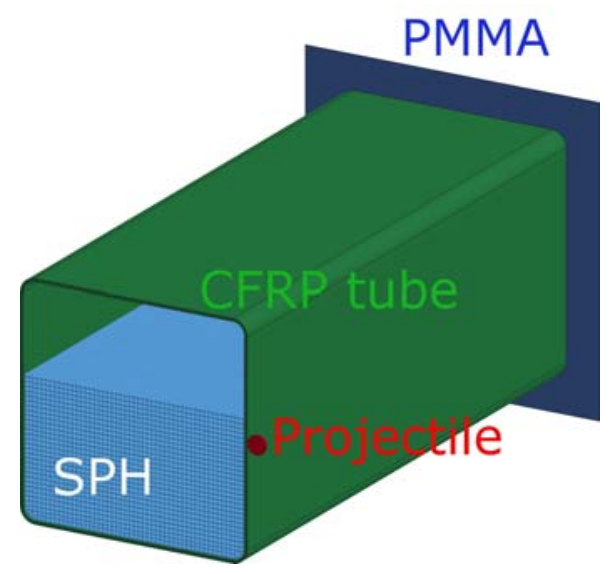

Fig. 3. SPH model done in LS-DYNA v.R7.

done through a penalty-based node-to-surface contact, where all SPH particles are considered as nodes that interact with the solid surface.

\subsection{Carbon fibre woven epoxy laminates model}

The behaviour of the carbon/epoxy woven laminate accounts for intra-laminar and inter-laminar damage. The former has been modelled as an orthotropic elastic material until failure, implemented through an user subroutine; whilst for the later a cohesive interaction is used. This kind of approach for intra-laminar damage has been widely used in impact problems on composite materials $[11,12]$. The use of cohesive behaviour for modelling the delamination can also be found in different works [13-17]. In the CFRP model, two failure mechanism are taken into account: fibre failure and matrix failure. Different failure variables $d_{i}$ (based on stresses) are defined for each failure mechanism. When the value of any of this variables reaches the value of 1 , failure initiates and all the components of the stress tensor $\sigma_{i j}$ involved in the failure definition are set to zero. In addition, a element removal criteria is used, based on total strain. The properties of the carbon fibre woven laminates are presented in table 2 . The mechanisms are: 
- Fibre failure. Due to woven configuration, fibre failure can appear in the two inplane axes. The fibre failure criteria are described by means of $d_{f 1}$ and $d_{f 2}$, one for each fibre direction:

$$
\begin{aligned}
& d_{f 1}=\left\{\begin{array}{lll}
\frac{\sigma_{11}}{X_{t}} & \text { if } & \sigma_{11}>0 \\
\frac{\left|\sigma_{11}\right|}{X_{c}} & \text { if } & \sigma_{11}<0
\end{array}\right. \\
& d_{f 2}=\left\{\begin{array}{lll}
\frac{\sigma_{22}}{Y_{t}} & \text { if } & \sigma_{22}>0 \\
\frac{\left|\sigma_{22}\right|}{Y_{c}} & \text { if } & \sigma_{22}<0
\end{array}\right.
\end{aligned}
$$

where $X_{t}$ and $X_{c}$ are the strengths of the composite laminate in tension and compression for the warp direction, and finally $Y_{t}$ and $Y_{c}$ are the strengths in tension and compression for the fill direction.

- Matrix failure. The matrix failure distinguished two mechanisms: in-plane shear $\left(d_{m 12}\right)$ and out-plane crushing $\left(d_{m 3}\right)$.

$$
\begin{aligned}
& d_{m 12}=\frac{\sigma_{12}}{S_{12}} \\
& d_{m 3}=\frac{1}{4}\left(\frac{\sigma_{33}}{Z_{c}}\right)^{2}+\frac{Z_{c} \cdot \sigma_{33}}{4 S_{13} S_{23}}+\left|\frac{\sigma_{33}}{Z_{c}}\right|+\max \left[\left(\frac{\sigma_{13}}{S_{13}}\right)^{2},\left(\frac{\sigma_{23}}{S_{23}}\right)^{2}\right]
\end{aligned}
$$

where $S_{12}, S_{13}$ and $S_{23}$ are the shear strengths in the three different planes and $Z_{c}$ is the strength in the through-thickness direction under compression. The equation 7 applies only when $\sigma_{33}<0$.

Inter-laminar damage is modelled through a cohesive surface interaction. The cohesive behavior is based on a traction-separation law, in which is necessary to define a damage initiation criteria and a damage evolution law. In this work, the damage initiation is defined by eq. 8 , where $\delta^{0}$ is the displacement at which softening starts, 


\section{Elastic properties}

\begin{tabular}{cccccc}
\hline$E_{1}=E_{2}$ & $E_{3}$ & $\nu_{12}$ & $\nu_{13}=\nu_{23}$ & $G_{12}$ & $G_{23}=G_{13}$ \\
$68 \mathrm{GPa}$ & $10 \mathrm{GPa}$ & 0.22 & 0.49 & $5 \mathrm{GPa}$ & $4.5 \mathrm{GPa}$ \\
\hline \hline Strength properties & & & & & \\
\hline$X_{t}=Y_{t}=X_{c}=Y_{c}$ & $Z_{c}$ & $Z_{r}$ & $S_{12}$ & $S_{13}$ & $S_{23}$ \\
$880 \mathrm{MPa}$ & $340 \mathrm{MPa}$ & $96 \mathrm{MPa}$ & $84 \mathrm{MPa}$ & $120 \mathrm{MPa}$ & $120 \mathrm{MPa}$ \\
\hline \hline Maximum strain & & & & & \\
\hline$\varepsilon_{1}=\varepsilon_{2}$ & $\varepsilon_{3}$ & $\varepsilon_{12}=\varepsilon_{23}=\varepsilon_{13}$ & & & \\
0.025 & 0.05 & 0.1 & & &
\end{tabular}

\section{Table 2}

Properties of woven carbon/epoxy laminate.

$E_{N}$ and $E_{T}$ are the stiffness normal to the plane of cohesive interaction and in tangential direction, respectively, $T$ and $S$ are the peak traction normal of the plane of cohesive interaction and in tangential direction, and finally $\beta$ is the ratio between displacement in mode II and I $\left(\beta=\delta_{I I} / \delta_{I}\right)$. The damage evolution is a potential law type based on energies, eq. 9 , where the ultimate displacement $\left(\delta^{F}\right)$ is defined as function of $G_{I C}$ and $G_{I I C}$, the energy release rate for mode I and II respectively; and the power law coefficient $(\mu)$. The properties used for the cohesive interaction can be seen in table 3 .

$$
\begin{aligned}
& \delta^{0}=\frac{T}{E_{N}} \frac{S}{E_{T}} \sqrt{\frac{1+\beta^{2}}{\left(\frac{S}{E_{T}}\right)^{2}+\left(\beta \frac{T}{E_{N}}\right)^{2}}} \\
& \delta^{F}=\frac{2(1+\beta)^{2}}{\delta^{0}}\left[\left(\frac{E_{N}}{G_{I C}}\right)^{\mu}+\left(\frac{E_{T} \cdot \beta^{2}}{G_{I I C}}\right)^{\mu}\right]^{-\frac{1}{\mu}}
\end{aligned}
$$




\begin{tabular}{lllllll}
\hline \hline$E_{N}$ & $E_{T}$ & $T$ & $S$ & $G_{I C}$ & $G_{I I C}$ & $\mu$ \\
\hline
\end{tabular}

$40 \mathrm{GPa} \quad 30 \mathrm{GPa} \quad 11 \mathrm{MPa} \quad 45 \mathrm{MPa} \quad 287 \mathrm{~J} / \mathrm{m}^{2} \quad 1830 \mathrm{~J} / \mathrm{m}^{2} \quad 1.42$

\section{Table 3}

Parameters for the cohesive interface.

\subsection{Box and projectile lagrangian FE model}

The woven CFRP tube is discretised by means of eight node linear solid elements with reduced integration and hourglass control. The mesh is more refined in the impacted zone $\left(1 \times 1 \mathrm{~mm}^{2}\right)$ than far from the impact point (until $\left.7.3 \times 4.5 \mathrm{~mm}^{2}\right)$, obtaining a mesh that accurately reproduces the damage induced according to previous works [18]. The CFRP tube walls present 10 elements through thickness, so each element corresponds to one ply. The projectile is discretised by means of eight node conventional solid elements with reduced integration and modelled as an elastic material $\left(\rho=7850 \mathrm{Kg} / \mathrm{m}^{3} ; E=210 \mathrm{GPa} ; \nu=0.3\right.$ ). The PMMA window is discretised by means of four node conventional shell elements with reduced integration and modelled as an elastic material $\left(\rho=1180 \mathrm{Kg} / \mathrm{m}^{3} ; E=3 \mathrm{GPa} ; \nu=0.35\right)$. Both, steel and PMMA, are modeled as elastic materials since no plastic deformation nor damage is observed in none of them in the experimental tests.

\section{Results}

Numerical simulations were carried out at different impact velocities $(600 \mathrm{~m} / \mathrm{s}$ and $900 \mathrm{~m} / \mathrm{s}$ ) and different fluid filling levels $(60 \%$ and $75 \%)$. The results obtained are compared with the experimental data available [6] in order to validate the numerical model. Additionally, MM-ALE method and SPH are compared analyzing their advantages and disadvantages to reproduce the effects of the HRAM phenomenon in partially fluid filled CFRP tubes. Moreover, the kinematics of the layer of fluid that 
initially is above the projectile trajectory will be studied in detail. This fluid layer is raised by the projectile impacting the upper wall of the CFRP tank. A complete analysis of this fluid-structure interaction is done in the section 4.2 .

\subsection{Validation}

The numerical results are compared qualitative and quantitatively to the experimental data.

- Projectile position. The experimental projectile position, obtained by means of a high-speed camera, can be compared with the numerical data. The experimental and numerical time history of the projectile is depicted in Fig. 4. It is observed a good correlation between the curves, for both MM-ALE method and SPH. The trend of the projectile velocity can be observed by means of the projectile position slope. The velocity decreases inside the fluid, transforming part of its kinetic energy into pressure and kinetic energy in the fluid. The projectile position inside the fluid is not affected for the fluid filled volume, Fig. 4 (a) and (b), so neither the energy transfer between fluid and projectile.

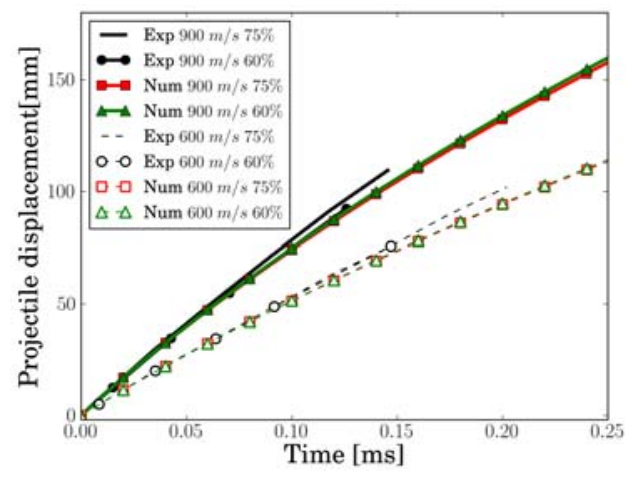

(a) MM-ALE

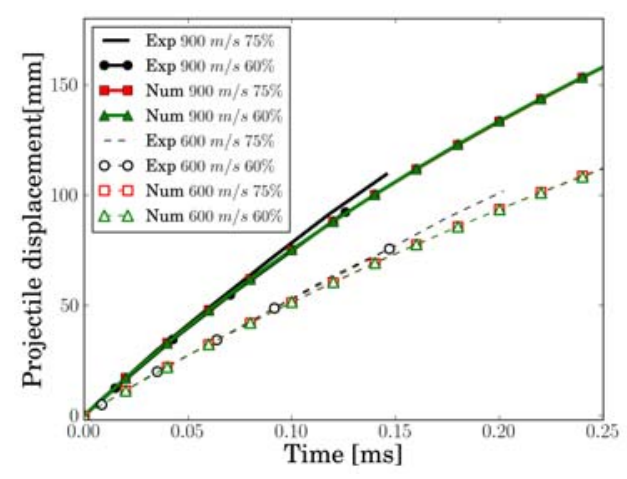

(b) $\mathrm{SPH}$

Fig. 4. Projectile position from experimental tests and numerical model

- HRAM phenomenon. Fig. 5 shows images of the penetration process when a 
projectile impacts at $600 \mathrm{~m} / \mathrm{s}$ into a tank filled at $60 \%$, both experimental and numerical. For a better representation of the phenomenon in the numerical simulations, the pressure contours are depicted. It can be seen how the numerical approaches, MM-ALE and SPH, qualitatively reproduce the main characteristics of an HRAM event. At $t=26 \mu s$ an hemispherical pressure wave generated by the impact is clearly shown. This pressure wave travels at the sound velocity through the fluid, while the drag force decelerates the projectile creating a cavity in the wake and an overpressure just ahead the projectile, as it is seen at $t=83 \mu \mathrm{s}$. The cavity grows and pushes the layer of fluid that initially is above the projectile trajectory, $t=249 \mu s$; in addition the overpressure of the fluid ahead the projectile will cause a pre-stress situation in the exit wall that will generate a higher damage than in the entry wall. Finally, the cavity continues growing and the layer of fluid approaches the upper wall, $t=472 \mu s$.

Although the same comparison can be done in all the considered cases, it is worth to mention a difference between the $75 \%$ and the $60 \%$ cases. The angle of the layer of fluid with respect to the horizontal is higher in the case of $60 \%$ than in the case of $75 \%$. This can be explained by the fact that the energy transferred by the projectile to the fluid in both cases is the same, as it has been shown by means of the projectile position history. Therefore when the mass of the layer of fluid is smaller (60\% case) it travels faster that when the mass is higher.

- Pressure field. The experimental pressure data in two different points of the fluid (PTn, near the impact point and PTf, far form impact point) is compared with the numerical results. The pressure values obtained in the pressure transducers, both experimental and numerically (MM-ALE and SPH), when a $75 \%$ partially fluid filled tube is impacted at $900 \mathrm{~m} / \mathrm{s}$ are depicted as an example in Fig. 6. A good correlation can be observed for both numerical approaches not only in peak values but in the trends regarding pressure transducer location influence. It can be noticed that SPH pressure values are slightly slower, this is probably due to 

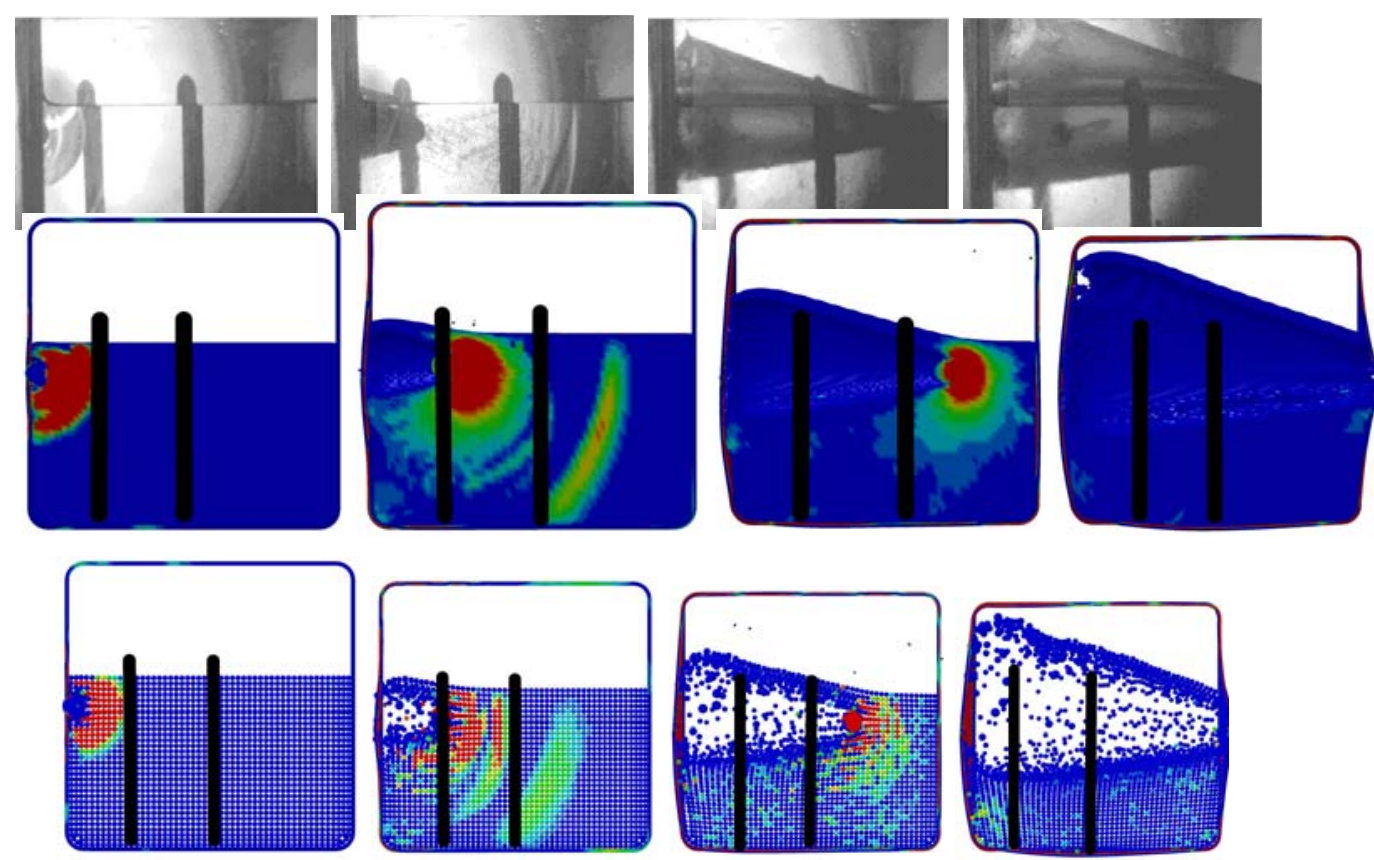
(a) $26 \mu \mathrm{s}$
(b) $83 \mu \mathrm{s}$
(c) $249 \mu \mathrm{s}$
(d) $472 \mu \mathrm{s}$

Fig. 5. Impact process of a projectile impacted at $600 \mathrm{~m} / \mathrm{s}$ into a tank filled at $60 \%$.

Top: Experimental images. Middle: Pressure contour in MM-ALE numerical model.

Bottom: Pressure contour in SPH numerical model. Pressure contour, ranging from red $10 M P a$ to blue $0 M P a$

the fact that the MM-ALE mesh is finer than SPH discretization. Similar results are obtained for the other cases not presented here.

Failure in tubes. The failures that appear numerically in the CFRP tubes are compared qualitatively with the experimental results. Fig. 7 shows the main failures, which appear on the upper and exit walls, of a partially filled CFRP tank impacted at $900 \mathrm{~m} / \mathrm{s}$. It can be seen that the exit wall presents a cross-type failure that is well predicted by the numerical approaches for both fluid filled levels. The failure on the upper wall is due to the impact of the layer of fluid that is raised by the projectile; it begins in the border of the exit wall and it advances through the upper wall. This kind of failure only appears in partially filled cases and is higher for the case of $75 \%$ than in $60 \%$. Both numerical methods reproduce the 


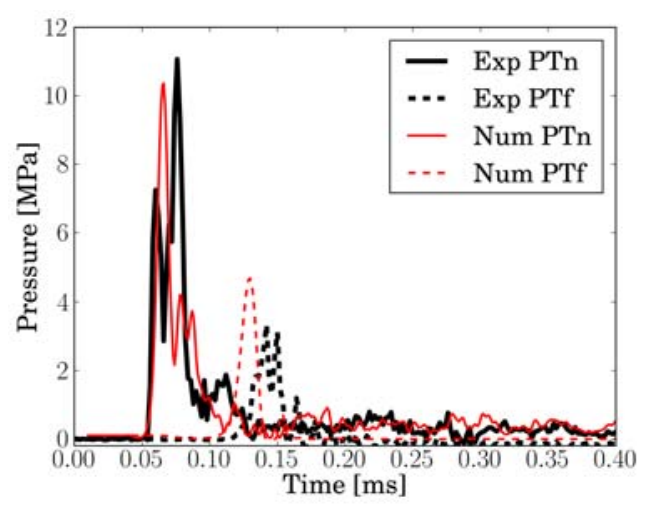

(a) MM-ALE

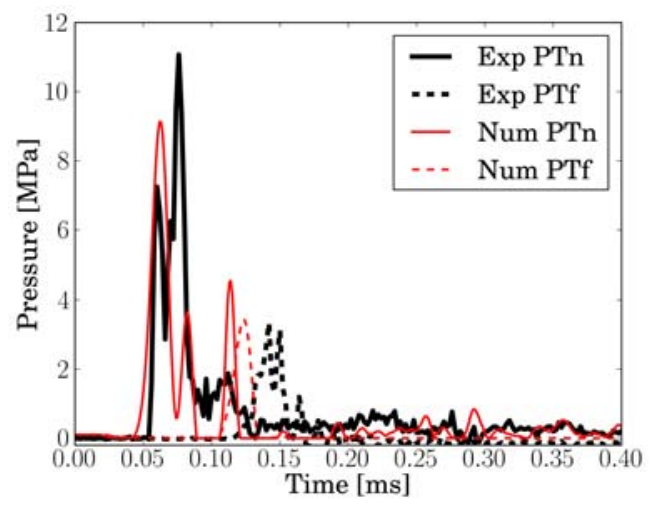

(b) $\mathrm{SPH}$

Fig. 6. Pressure values registered from experimental tests and numerical model in the impact at $900 \mathrm{~m} / \mathrm{s}$ with $75 \%$.

failure on the border and its length but not the propagation through the wall, so a deeper analysis of this will be done in the next section. However, despite the complexity of this process and that the influence of CFRP manufacturing in the tube behavior makes difficult to reproduce same experimental failures in numerical simulation, similar trends can be seen in both experimental and numerical final failures.
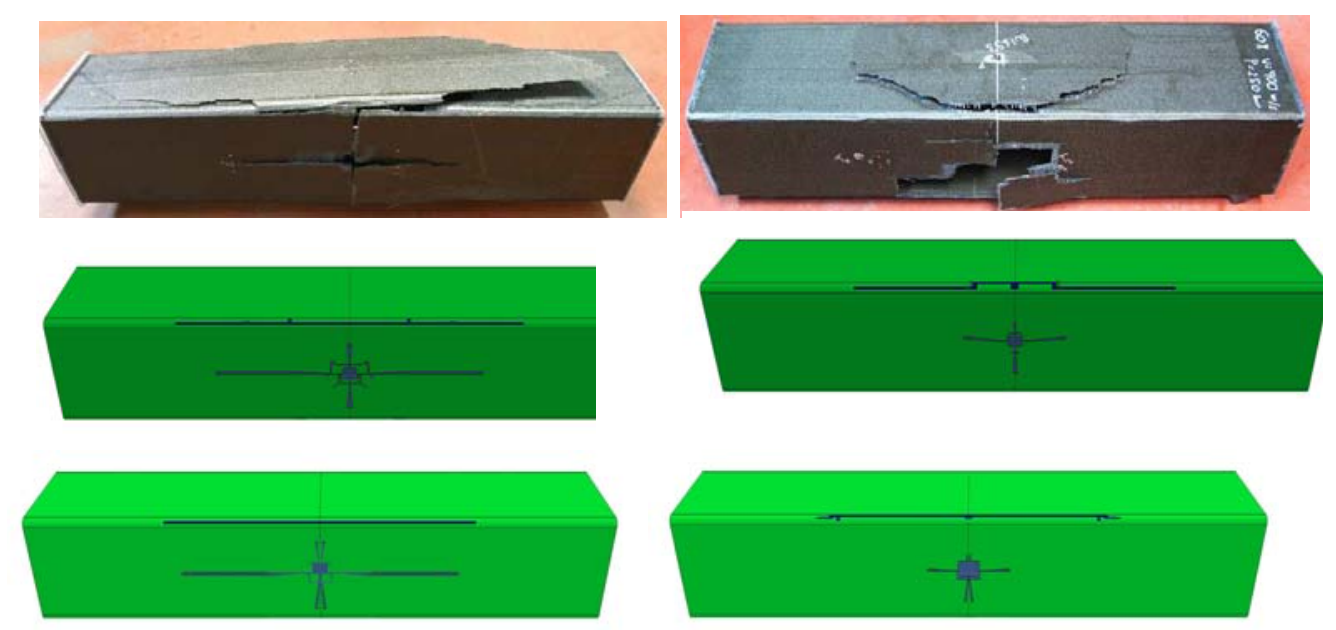

(a) $75 \%$

(b) $60 \%$

Fig. 7. Failure on upper and exit walls of CFRP tube impacted at $900 \mathrm{~m} / \mathrm{s}$. Top: Experimental. Middle: MM-ALE. Bottom: SPH 
In view of the results, it can be concluded that both numerical approaches (MMALE and SPH), and the material model implemented can reproduce faithfully the HRAM phenomenon and its effects in a partially filled fluid CFRP tank. There are no differences in the results to identify which is the best method. However, the computational cost of the MM-ALE model is much lower than the SPH one (both cases were run in the same workstation, Intel(R) Xeon(R) E5645, in which simulation lasted 72 and 186 hours respectively, using 12 cores). Therefore, it can be said that the MM-ALE is the preferable approach for the simulation of this kind of problems.

\subsection{Analysis of the fluid-structure interaction in the upper wall}

The HRAM phenomenon has been usually analyzed according the dangerous effects that it causes in the structure of completely filled containers. When the HRAM phenomenon occurs in a partially filled fluid tank, the process of impact of the layer of fluid into the structure and its effects have to be taken into account and analyzed. In this section the results obtained in the MM-ALE model, previously validated, are taken into advantage to study the fluid-structure interaction in the wall which initially is not in contact with the water (upper wall). A detailed analysis of the kinetic energy transferred between the projectile and the fluid, and the fluid and the upper wall is performed to try to understand how this process influences the failure of the upper wall.

Fig. 8 shows the kinetic energy of the water and the upper wall for an impact at $900 \mathrm{~m} / \mathrm{s}$ and partially filled conditions $(60 \%$ and $75 \%)$. The different instants denoted with letters inside the images correspond to the images shown in Fig. 9. It can be seen that the kinetic energy of the fluid increases while the projectile is inside the tank transferring its energy. The transfer of energy finishes when the 
projectile impacts the exit wall, reaching the maximum kinetic energy of the fluid at $t=240 \mu s$ after the impact, Fig. 9 (a) and (d). As it was already shown in Fig. 4 the position of the projectile is not influenced by the volume in the cases under study, therefore the projectile impacts the exit wall at the same time (Fig. 9 (a) and (d)) and the energy transferred to the fluid is the same for both fluid filled levels (Fig. 8 left). This is the reason why the layer of fluid above the projectile raises faster in the $60 \%$ filled case and impacts before the upper wall, Fig. 9 (b) and (e). After the layer of fluid reaches the upper wall, the kinetic energy of the fluid decreases more strongly, while the kinetic energy of the upper wall increases suddenly due to the violent shake, Fig. 8 right (instants (b) and (e)). When the whole layer of fluid is in contact with the upper wall pushing it, Fig. 9 (c) and (f), the maximum kinetic energy of the upper wall is reached, Fig. 8 right (instants (c) and (f)). The layer of fluid continues pushing the upper wall and $100 \mu s$ later appears the failure in the border of the upper wall for both partially fluid filled cases, as happened in the experimental tests. A detail of that failure is depicted in Fig. 10 (a) and (b). The fluid continues pushing the upper wall towards the longest dimension of the tube transferring its kinetic energy. In Fig. 8 right can be observed that the kinetic energy of the upper wall in the $75 \%$ case is higher during a longer time than in the case of $60 \%$, this could be the reason why the failure is higher in the former case. Although the physical phenomenon of the event seems to be well reproduced and explained by the model, the propagation through the upper wall observed in the experimental tests is not captured. Maybe, a more sophisticated material model could improve the results regarding the progression of the failure. In addition it has to be mentioned that the influence of the manufacturing of the CFRP tubes may be affecting their behavior. Finally, a detail of the maximum delamination reached in the upper wall is shown in Fig. 10 (c) and (d). It is observed that delamination in the $60 \%$ case is higher than in the $75 \%$ case, opposite to failure. This means that a sudden impact at high velocity can induce the same or even more delamination 
than an impact at a slower velocity and more mass, which does not mean that the final failure will be lower.

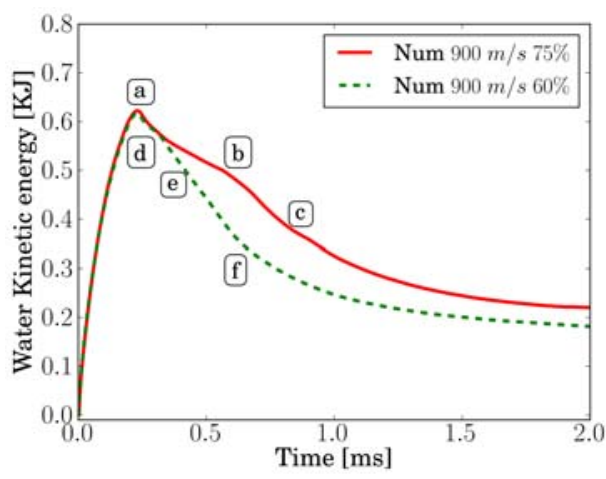

(a) Fluid kinetic energy

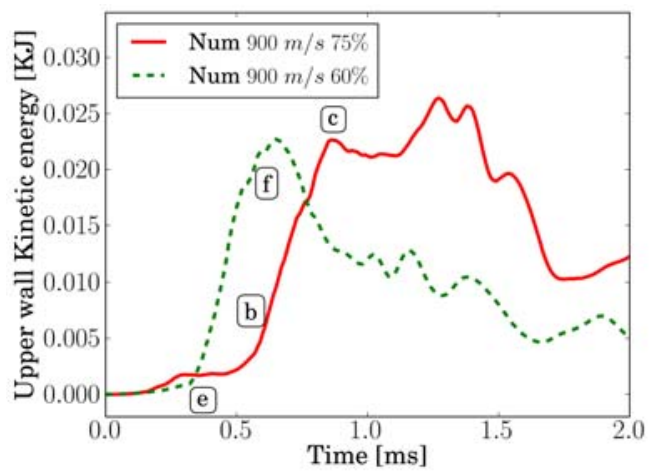

(b) Upper wall kinetic energy

Fig. 8. Kinetic energies for the impact at $900 \mathrm{~m} / \mathrm{s}$ taken from the MM-ALE model

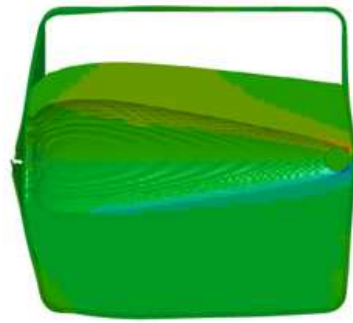

(a) $75 \%, 240 \mu \mathrm{s}$

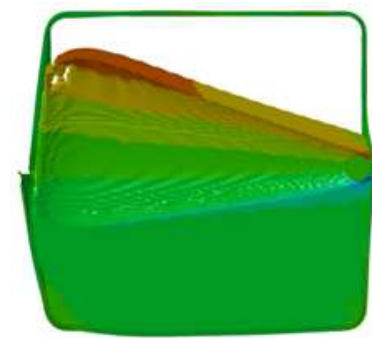

(d) $60 \%, 240 \mu \mathrm{s}$

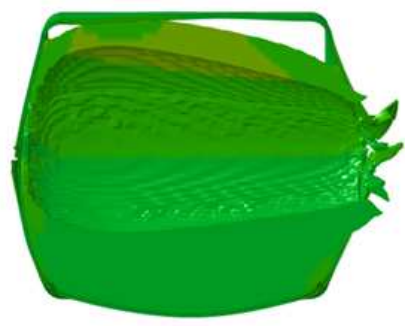

(b) $75 \%, 600 \mu \mathrm{s}$

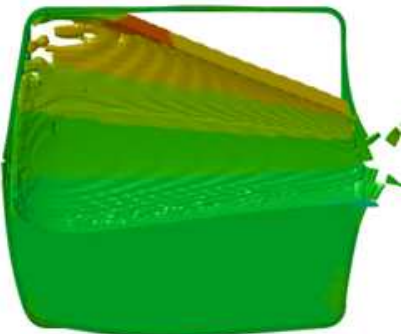

(e) $60 \%, 350 \mu \mathrm{s}$

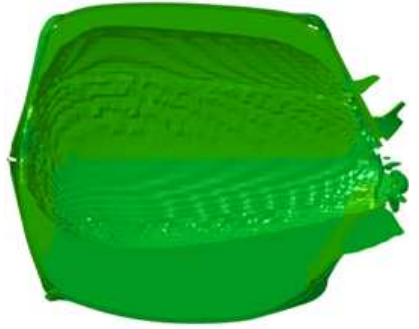

(c) $75 \%, 850 \mu \mathrm{s}$

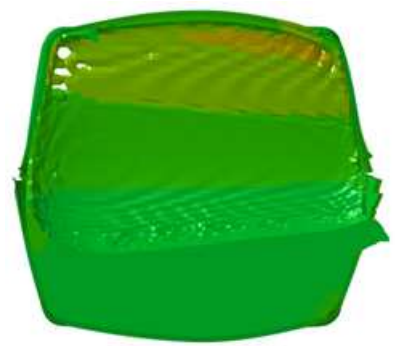

(f) $60 \%, 600 \mu \mathrm{s}$

Fig. 9. Vertical velocity contours, ranging from red $300 \mathrm{~m} / \mathrm{s}$ to blue $-300 \mathrm{~m} / \mathrm{s}$, taken from the MM-ALE model. Top: $75 \%$ filled case impacted at $900 \mathrm{~m} / \mathrm{s}$. Bottom: $60 \%$ filled case impacted at $900 \mathrm{~m} / \mathrm{s}$ 


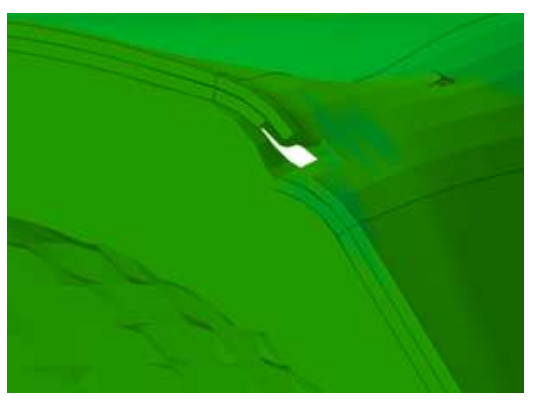

(a) $75 \%, 1000 \mu \mathrm{s}$

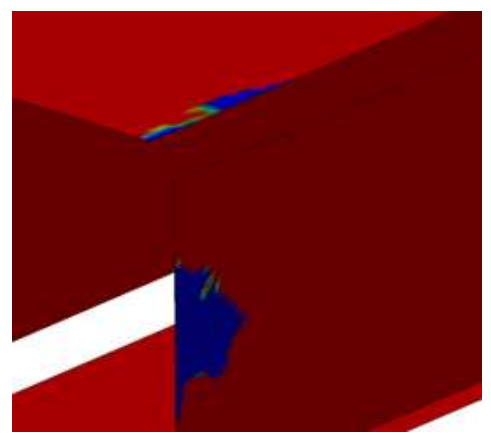

(c) $75 \%, 1200 \mu \mathrm{s}$

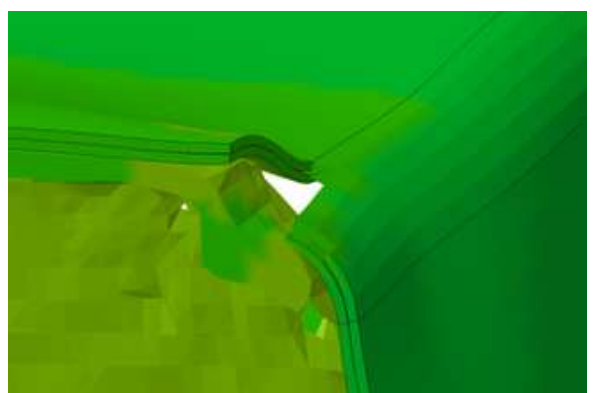

(b) $60 \%, 700 \mu \mathrm{s}$

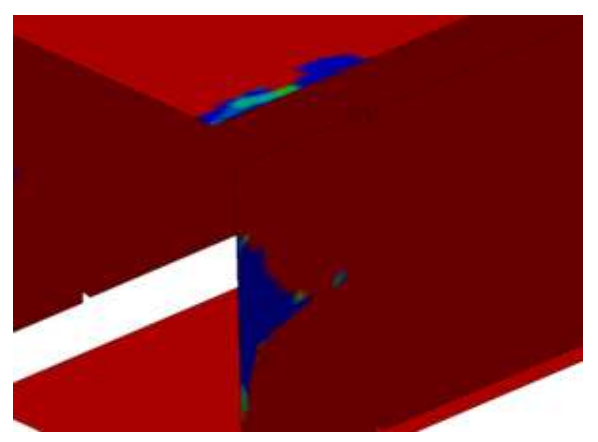

(d) $60 \%, 900 \mu \mathrm{s}$

Fig. 10. Numerical images taken from the MM-ALE model at $900 \mathrm{~m} / \mathrm{s}$ Top: Detail of the onset failure in the upper wall. Bottom: delamination, ranging from red (fully damaged) to blue (no damage).

\section{Conclusions}

In this work, two different numerical approaches, MM-ALE and SPH, have been used to try to reproduced, analyze and understand the special events that happen due to the HRAM phenomenon in a partially filled fluid CFRP tube. Certain conclusions can be remarked:

- MM-ALE and SPH implemented in LS-DYNA v.R7 have been shown as reliable tools to reproduce HRAM phenomenon in partially filled cases. Both methods predict faithfully the energy loss by the projectile, the pressure values registered in the fluid, and in a qualitatively way, the overall process of the HRAM phenomenon. Due to the high computational costs of the SPH method, MM-ALE is the 
suggested approach in this kind of problems.

- The CFRP model implemented using a user subroutine and a cohesive interaction reproduces the main experimental failures that appear in partially filled fluid tubes subjected to impact. The cross-shaped failure that appears in the exit wall is well predicted. The trends and the length regarding the failure of the wall which initially is not in contact with the water (upper wall) and that only appears in partially fluid filled cases impacted at $900 \mathrm{~m} / \mathrm{s}$, are well predicted, whereas the progression of the failure in that wall is not adequately reproduced.

- The impact process of a layer of fluid raised by the projectile movement inside a partially filled tank has been analyzed in order to understand its importance and influence in the failure effects of the CFRP structure. It has been shown that the main responsible of the upper wall failure is the kinetic energy of that layer of fluid and that it has to be taken into account for survivability designs.

\section{Acknowledgements}

This research was done with the financial support of the Spanish Ministry of Education under Project reference DPI2010-15123 and of the Region of Madrid and University Carlos III of Madrid under Project reference CCG10-UC3M/DPI-4694.

\section{References}

[1] López-Puente J, Zaera R, Navarro C. An analytical model for high velocity impacts on thin CFRPs woven laminates. Int J Solids Struct 2007;44:2837-51.

[2] Airoldi A, Cacchione B. Modelling of impact forces and pressures in lagrangian bird strike analyses. Int J Impact Eng 2006;32:1651-77. 
[3] Anghileri M, Castelleti LML, Invernizzi F, Mascheroni M. A survey of numerical models for hail impact analysis. Int J Impact Eng 2005;31:929-44.

[4] Pernas-Sánchez J, Pedroche DA, Varas D, López-Puente J, Zaera R. Numerical modeling of ice behavior under high velocity impacts. Int J Solids Struct 2012;49(14):1919-27.

[5] Mines RAW, McKown S, Birch RS. Impact of aircraft rubber tyre fragments on aluminium alloy plates: I-experimental. Int J Impact Eng 2007;34:627-46.

[6] Varas D, Zaera R, López-Puente J. Experimental study of fluid-filled tubes subjected to high-velocity impact. Compos Struct 2011;93(10):2598-609

[7] Artero-Guerrero JA, Pernas-Sánchez J ,Varas D, López-Puente J. Numerical Analysis of CFRP fluid-filled tubes subjected to high velocity impact. Compos Struct 2013;96:286-97

[8] Varas D, Zaera R, López-Puente J. Numerical modelling of the Hydrodynamic Ram phenomenon. Int J Impact Eng 2009;36:363-74.

[9] Boyd R, Royles R, El-Deeb KMM. Simulation and validation of UNDEX phenomena relating to axisymmetric structures. Sixth international LS-DYNA users conference similation. Dearborn (Michigan). April 2000.

[10] Hallquist JO. LS-DYNA Theory Manual. Livermore Software Technology Company. March 2006.

[11] Hou JP, Petrinic N, Ruiz C, Hallett SR. Prediction of impact damage in composite plates. Compos Sci Technol 2000;60(2):273-81.

[12] López-Puente J, Zaera R, Navarro C. Experimental and numerical analysis of normal and oblique ballistic impacts on thin carbon/epoxy woven laminates. Compos Part A-Appl S 2008;39:374-87. 
[13] Camanho PP, Davila CG. Mixed-Mode decohesion finite elements for the simulation of delamination in composite materials. Tech Report NASA/TM2002-211737 2002:1-37.

[14] Turon A, Camanho PP, Costa J, Renart J. Accurate simulation of delamination growth under mixed-mode loading using cohesive elements: Definition of interlaminar strengths and elastic stiffness. Compos Struct 2010;92(8):1857-64.

[15] Shi Y, Swait T, Soutis C. Modelling damage evolution in composite laminates subjected to low velocity impact. Compos Struct 2012;94(9):2902-13.

[16] González EV, Maimí P, Camanho PP, Turon A, Mayugo JA. Simulation of drop-weight impact and compression after impact tests on composite laminates. Compos Struct 2012;94(11):3364-78.

[17] Varas D, Artero-Guerrero JA, Pernas-Sánchez J, López-Puente J. Analysis of high velocity impacts of steel cylinders on thin carbon/epoxy woven laminates. Compos Struct 2013;95:623-29

[18] López-Puente J, Zaera R, Navarro C. High energy impact on woven laminate. J Phys IV 2003;110:639-44. 